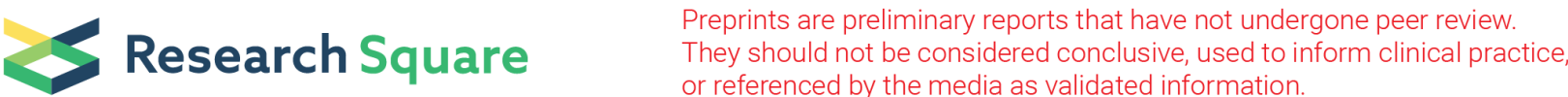

\section{Clinical Manifestations, Mutational Analysis, and Immunological Phenotype in Patients With RAG1/2 Mutations: First Cases Series From Mexico and Description of Two Novel Mutations.}

Mario Ernesto Cruz-Munoz ( $\square$ mario.cruz@uaem.mx ) Universidad Autonoma del Estado de Morelos https://orcid.org/0000-0001-6851-708X

Saul Oswaldo Lugo-Reyes

Instituto Nacional de Pediatría

Nina Pastor

Instituto Nacional de Pediatría

Edith González-Serrano

Instituto Nacional de Pediatría

Marco Yamazaki-Nakashimada

Instituto Nacional de Pediatría

Selma Scheffer-Mendoza

Instituto Nacional de Pediatría

Laura Berron-Ruiz

Instituto Nacional de Pediatría

Guillermo Wakida

Instituto Nacional de Pediatría

Maria Enriqueta Nuñez-Nuñez

Instituto Nacional de Pediatría

Ana Paola Macias-Robles

Centro Medico Nacional de Oriente IMSS

Aide Tamara Staines-Boone

Instituto Instituto Mexicano del Seguro Social

Edna Venegas-Montaya

Instituto Instituto Mexicano del Seguro Social

Carmen Alaez-Verson

Instituto Nacional de Medicina Genómica

Carolina Molina-Garay

Instituto Nacional de Medicina Genómica

Luis Leonardo Flores-Lagunes

Instituto Nacional de Medicina Genómica 


\section{Karol Carrillo-Sánchez}

Instituto Nacional de Medicina Genómica

Julie Niemela

National Institute of Health

\section{Sergio Rozensweig}

National Institute of Health

\section{Paul Gaytan}

Universidad Nacional Autónoma de México

Jorge Yañez

Universidad Nacional Autónoma de México

Ivan Martinez-Duncker

Universidad Autónoma del Estado de Morelos

\section{Luigi Notarangelo}

National Institute of Health

\section{Sara Espinosa-Padilla}

Universidad Autónoma del Estado de Morelos

\section{Research Article}

Keywords: Primary Immunodeficiencies, RAG1/2, T lymphocytes, SCID, Omenn Syndrome

Posted Date: February 17th, 2021

DOI: https://doi.org/10.21203/rs.3.rs-202390/v1

License: (c) (1) This work is licensed under a Creative Commons Attribution 4.0 International License. Read Full License 


\section{Abstract}

Mutations in Recombinase Activating Genes 1 and 2 (RAG1/2) results in human severe combined immunodeficiency (SCID). The products of these genes, are essential for $\mathrm{V}(\mathrm{D}) \mathrm{J}$ rearrangement of the antigen receptors during lymphocyte development. Nonsense mutations in RAG1 or RAG2 are associated with the most severe clinical and immunological phenotypes, whereas patients with missense mutations may develop leaky SCID or Omenn syndrome (OS). A group of non-previously recognized clinical phenotypes associated with granulomata and/or autoimmunity have been described as a consequence of hypomorphic mutations. Here we present six patients from unrelated families with missense variants in RAG1 or RAG2. Phenotypes observed in these patients ranged from OS to severe mycobacterial infections and granulomatous disease. Moreover, we report the first evidence of two previously unidentified variants as causative of pathological manifestations associated to immunodeficiency. This study represents the first case series of RAG1 or RAG2 deficient patients from Mexico and Latin America.

\section{Introduction}

Human severe combined immunodeficiency (SCID) comprises a group of inherited disorders that results from mutations in single-genes that govern lymphocyte development, differentiation, and/or function (1, 2). Until date, a group of at least 20 genes have been identified and characterized (3). Mutations in Recombinase Activating Genes 1 and 2 (RAG1/2), located in the human chromosome 11p13, represent approximately $10 \%$ of all SCID patients (4). The products of these genes, RAG1 and RAG2 proteins, are essential for $\mathrm{V}(\mathrm{D}) \mathrm{J}$ rearrangement of the antigen receptors during lymphocyte development. Since the first deleterious mutation in RAG genes identified by Schwarz in 1996 (5), more than 200 variants have been described in human patients. The clinical severity partially depends on the level of impairment in the recombinase activity of RAG1/2. Homozygous or compound heterozygous nonsense mutations in RAG1 or $R A G 2$ may result in null recombination activity and lead to the most severe clinical and immunological phenotypes, highlighted by the absence of $\mathrm{T}$ and $\mathrm{B}$ lymphocytes. In contrast, in patients carrying homozygous or compound heterozygous missense mutations, the recombinase activity is severely reduced but not absent, and patients may develop leaky SCID or its inflammatory variant, Omenn syndrome (OS).

A spectrum of less-severe clinical and immunological manifestations is now recognized, including combined immunodeficiency with granulomas, common variable immunodeficiency, autoimmunity and antibody deficiency (6-10). Moreover, these manifestations can appear early in childhood or during adolescence. These atypical SCID phenotypes have provided unique models to understand the genesis and mechanisms of autoimmunity and other immunological aspects. Here we present six patients from unrelated families with missense variants in RAG1 or RAG2. Phenotypes observed in these patients ranged from Omenn syndrome to severe mycobacterial infections and granulomatous disease. In addition, we report the first evidence of two previously unidentified variants as causative of pathological manifestations associated to immunodeficiency. 


\section{Methods}

Patients

Peripheral blood mononuclear cells (PBMC) were obtained from patients after securing informed consent forms approved by the local ethics committees in accordance with the declaration of Helsinki (Protocols 00165 and 049/2013). The study was multicentric and was conducted from January 2017 to December 2019.

\section{Mutations analyses at the RAG-1 and RAG-2 loci}

Genomic DNA was extracted from whole blood and analyzed via whole-exome sequencing (Illunina). All mutations were confirmed by Sanger sequencing. The RAG1 gene was amplified in 2 segments (94-1852 and 1781-3262), and the RAG2 gene was amplified in one segment (1201-2922). Sequencing was performed directly on the polymerase chain reaction (PCR) products by using the same set of primers.

\section{Protein modeling}

Structural models for the core regions of RAG1 and RAG2 are available in the Protein Data Bank (rcsb.org; (11)) for mouse (12-15), zebra fish $(16,17)$, and lancelet (18) proteins, with resolutions ranging from 2.75 to $4.6 \AA$. In order to determine whether the relative orientation of RAG1 and RAG2 changes depending on the type of RAG-DNA complex, each RAG1-RAG2 dimer was superimposed in VMD (19) using STAMP (20) in MultiSeq (21) against each RAG1-RAG2 dimer in the apo mouse RAG structure (PDB-ID 4WWX). The RAG1-RAG2 core human models were built in SwissModel (22), using structure 5ZDZ (chains A and B) as reference. As the mutations are located far from each other in the structure of the RAG1-RAG2 complex (Figure S1A and S1B), one multiple mutant model was constructed, containing all the mutations except RAG2-H481D. The RAG2-H481D mutation lies in the PHD domain at the C-terminal region of RAG2. Its structure was solved by X-ray crystallography (23), both as an apo form and in complex with modified histone-3 peptides. The wild type and mutant human PHD domains were built with SWISS-MODEL (22), using structure 2V87 (chain A) as template (Figure S1C). The models include residues 414-487 for RAG2. Hydrogen atoms were added in CHARMM-GUI (24), taking care that zinc-binding atoms had the appropriate protonation; the structures were energy-minimized in CHARMM45 $(25,26)$. The energyminimized structures were analyzed in RAMPAGE (27) to detect residues in forbidden regions of the Ramachandran plot.

\section{Clinical classification of the RAG1 and RAG2 variants.}

Variants were classified using the criteria in the Standards and Guidelines for the Interpretation of Sequence Variants developed by the American College of Medical Genetics and Genomics (ACMG) and the Association for Molecular Pathology (28). The information available in public databases and literature was also considered for the final classification of each variant. The effect of the mutation was evaluated using several algorithms: PolyPhen2 (29), SIFT (30), PredictSNP (31), VarSite (32), REVEL (33) and PON-P2 (34) (Table S1). Except for PolyPhen2 and SIFT, the others are consensus predictors, which 
use the output from individual programs as part of their training procedure to distinguish damaging from benign changes. ProTSPoM (35) and Missense3D (36) are strictly structural predictors, which analyze the effect of the mutation in the context of available or modeled structures, respectively.

\section{T lymphocytes subsets and Proliferation assays.}

Peripheral blood mononuclear cells (PBMC) were isolated using Ficoll density separation (GE healthcare, Life Systems). For the evaluation of different lymphocyte subsets, PBMCs were stained with the following fluorochrome-conjugated monoclonal antibodies against the following cell surface markers: CD3 FITC (Biolegend, clone OKT3), CD4 PE (Biolegend, clone OKT4), CD8 APC (Biolegend, clone SK1), CD19 PerCP-Cy5 (Biolegend, clone 4G7), CD56 APC (Biolegend, clone 5.1H11), CD45 RA PE (Biolegend, clone HI100), and CD45 RO APC (Biolegend, clone UCHL1). For proliferation assays, PBMCs were stained with CellTracker ${ }^{\mathrm{TM}}$ green CMDFA dye (ThermoFisher Scientific, USA). Cells were stimulated with purified anti-CD3 plus anti-CD28 antibodies (BioLegend, USA) or PHA (Sigma) for 5 days at $37^{\circ} \mathrm{C} 5 \% \mathrm{CO}_{2}$. Before to sample acquisition, PBMCs were stained using the murine PE-conjugated monoclonal antibody against human CD3 (Biolegend, clone OKT3). All samples were acquired on FACSCanto II (BD bioscience) and analyzed with the use of FlowJo 7.6.5 software (Tree Star, Ashland, OR).

\section{Results}

\section{Case 1}

Patient was a two-month-old girl born to consanguineous parents. She was referred to a local hospital for bilateral otorrhea, eczema and generalized seborrheic dermatitis. Family background included 3 dead siblings. Before hospitalization, the patient had received the BCG and the Hepatitis B vaccines, with no apparent complications. Upon admission, physical examination found abundant purulent discharge from the ears; generalized dermatitis with desquamation, erythema and pruritic maculopapular lesions. At age three months old, the patient presented bilateral purulent otic secretion, hepatosplenomegaly and diarrhea. At four months old, she developed rhinorrhea and cough, which persisted one month later despite oral antibiotics treatment. Treatment was started with monthly intravenous immunoglobulin (IVIG) replacement therapy and prophylaxis with trimethoprim/sulfamethoxazole (TMP/SMZ) and fluconazole. The patient died of HSCT-related complications. Immunological work-up revealed low to undetectable serum levels of immunoglobulins and lymphopenia (Table S2). A homozygous missense variant (rs762054841) in exon 2 of RAG2 (RAG NM_000536 c.104G>T, p. G35V), was revealed by targeted exome sequencing. This variant is classified as likely pathogenic according to the ACMG criteria and it has been reported previously (Clinvar Allele ID 488119) (37-41) (Table S3). RAG2-G35 lies in the first Kelch repeat (Figure S1B) at the center of a loop with very specific conformational requirements, which enable the interaction of RAG1-E669 with the peptide bond NH group of RAG2-G35 (Figure 1A). This conformation is buttressed by RAG2-Q33, which forms hydrogen bonds to two consecutive peptide bond carbonyls (broken black lines in Figure 1A). Mutation to V35 results in steric clashes within RAG2 and with RAG1 (Figure 1B, red broken lines). The sidechain of V35 bumps against the beta carbon of RAG1- 
H668, a clash that could be relieved by separating RAG1 from RAG2 but would debilitate the complex. Within RAG2, there are clashes with the backbone of V35, a consequence of this residue lying in a forbidden region of the Ramachandran plot. There is also a clash with the amino group of RAG2-K37, which can be eliminated by rotating this particular sidechain, as seen in some structures of RAG2 from zebra fish. Rotating the sidechain of V35 does not eliminate the clashes with the backbone of RAG2 and with RAG1-H668. The mutation can be found in healthy people, but at a very low frequency and as a heterozygote (42). Regarding the neighboring residues, mutations RAG2-Q33E and RAG1-H668Y are benign according to dbSNP (43) and gnomAD, whereas mutation of RAG1-E669 to K or G causes Omenn Syndrome or SCID, respectively (44).

\section{Case 2.}

Patient is a three-month old boy born to non-consanguineous parents who was referred to a local hospital for acute diarrhea. He has two healthy older siblings and tolerated the BCG vaccine at birth. Past medical history included eczema, allergy to cow's milk proteins, skin rash, adverse drug reactions, at least four previous hospitalizations to treat urinary tract infections, and sepsis. While hospitalized, patient developed bilateral otitis media, seborrheic dermatitis, severe anemia and recurrent upper respiratory tract infections. Laboratory work-up showed low levels of serum immunoglobulins and lymphopenia (Table $\mathrm{S} 2$ ). Exome sequencing revealed two missense variants in RAG2 (NM_000536.4): a missense variant consisting of a $\mathrm{T}$ to a $\mathrm{C}$ transition at position 464 of $R A G 2 \mathrm{cDNA}$ (c.464T $>\mathrm{C}$ ), causing a Leucine $(\mathrm{L})$ to Proline (P) change at position 155 (p.L155P), and a transition of $\mathrm{C}$ to $\mathrm{T}$ at position 685 of RAG2 cDNA (c.685C>T), causing an Arginine (R) to a Tryptophan ( $T$ ) change at position 229 (p.R229W), rs765298019. L155P is predicted likely pathogenic according to ACMG, it has been reported once in the ClinVar database (Variation ID: 418452, as of august 2020), and classified as likely pathogenic but the associated phenotype was not specified. R229W is classified as likely pathogenic according to ACMG criteria, and it is has been reported several times in association to RAG2 deficiency $(39,40,45-49)$ and Table S3.

RAG2-L155 lies at the core of the third Kelch repeat in RAG2 (Figure S1B), snugly fitting into a hydrophobic and aromatic box, connecting with its sidechain the beta sheet of its repeat with that of the previous one by interacting with RAG2-H94 (Figure 2A). Upon mutation to P, many of these interactions are lost, including (Figure 2B). This repeat includes the longest loop in RAG2, protruding towards RAG1 in the complex (Figure S1A, RAG1-loop), and changes in the packing of residue 155 could alter either the conformation or the position of this loop, affecting the interaction with RAG1. This variant has not been associated with OS or SCID, and can be tolerated as heterozygous (gnomAD). On the other hand, the double mutant V154A/L155A is inactive (50), suggesting that the packing of this Kelch repeat is important for function. RAG2-R229 lies at the border between two Kelch repeats (repeat 4 and 5, Figure S1B) engaging in aromatic interactions with RAG2-H222 and a salt bridge with RAG2-E280, and also forms a salt bridge with RAG1-D549 (Figure 3A). It appears, therefore, important for the structural stability of RAG2 and also for interacting with RAG1. Mutation to W eliminates the possibility of salt bridges, and 
also increases the distance to RAG1-D549 (Figure 3B). This mutation was described for three homozygous patients (49), but no activity assay has been published yet.

\section{Cases $3 \& 4$}

Patient number 3 is a teenage male, born to consanguineous parents. The patient was referred to local hospital for a history of recurrent infections, starting before one year old. Perinatal history had been uneventful; he received the BCG vaccine at birth, with no adverse reactions. Infections included: respiratory, gastrointestinal, oral candidiasis, and herpetic stomatitis. At age 4, after multiple antibiotic treatments, he underwent tympanic ventilation tubes placement and adenotonsillectomy. He also developed mild anemia and bronchiectasis as complications. At age 10, he suffered from ganglionar tuberculosis, for which he received a four-drug antimycobacterial regimen for 3 months (maintenance for a year with 2 drugs). At age of 12, he developed gallstones and underwent cholecystectomy; and at age 16 , he was treated for chronic diarrhea and bona-fide abdominal tuberculosis. On physical examination he had growth failure and malnutrition, with a body mass index below the fifth percentile, non-palpable lymph nodes, and bilateral cracking rales on chest auscultation. High-resolution chest CT scan confirmed extensive bilateral bronchiectasis. Laboratory work-up showed low serum levels of IgG subclasses and persistent lymphopenia (Table S2 and Figure 4A). As shown in figure 4B, the CFSE lymphocyte proliferation assay in response to PMA/ionomycin, PHA and anti-CD3/CD28 was impaired after 5 days compared to healthy control. He was treated since age 13 with intravenous immunoglobulin (IVIG) and oral antibiotic (trimethoprim/sulfamethoxazole and itraconazole) prophylaxis. The patient is alive but has been lost for follow up.

Patient number 4 is a six-year-old girl with chronic lung disease and skin ulcers who was referred to local hospital for suspected SCID in 2012. Her parents are unrelated from patient number 3 . She has three healthy siblings and received the BCG vaccine at birth, with no apparent adverse reactions. She started at age 4 , with chronic dry cough in bouts, and a feeling of weakness. At age 5 , she developed an erythematous papular lesion in one of her fingers. She was first hospitalized for pneumonia at age 6 . Papules had turned into oval-shaped ulcers 10 to $50 \mathrm{~mm}$ in diameter, covering her right arm, face, both legs, and buttocks, over the course of a year; she also developed discolored disseminated lesions typical of vitiligo in her eyelids and chest. Other manifestations included an eye granuloma, herpetic stomatitis, pansinusitis, and bilateral bronchiectasis. Physical examination confirmed skin lesions including discolored patches, round ulcers and scars; as well as short stature, undernutrition and a temperature of $38^{\circ} \mathrm{C}$, with mild polypnea, dental cavities, bilateral non-tender cervical lymphadenopathy of around $15 \mathrm{~mm}$, and bilateral rhonchi and rales on auscultation. A high-resolution tomography of the chest showed small bronchiectasis and diffuse interstitial fibrosis suggestive of bronchiolitis. For the skin ulcers, differential diagnoses of sporotrichosis, skin tuberculosis, and granulomatous vasculitis were all entertained. Workup for mycobacteria was persistently negative, but skin biopsies reported necrotizing granulomatous interface dermatitis with micro-abscesses. She was diagnosed with bona-fide skin tuberculosis, vitiligo, pansinusitis and bronchiolitis obliterans, and started treatment with a 4-drug oral antibiotic regime, intravenous gammaglobulin and supplementary oxygen overnight, with a provisional 
diagnosis of Combined Immunodeficiency. She was hospitalized for pneumonia and died of refractory septic shock. Blood counts reported low serum immunoglobulin levels and lymphopenia (Table S2). Flow cytometry for lymphocyte subsets showed a T-B-NK ${ }^{+}$phenotype (Figure 4A). Lymphoproliferation was impaired for PMA/ionomycin, phytohemagglutinin and anti-CD3 plus anti-CD28 stimuli (Fig 4B).

For patients 3 and 4, targeted exome sequencing revealed the same homozygous missense mutation in exon 3 of RAG2(NM_000536.4):c.1441C>G, (p.His481Asp) (rs762054841), a rare variant affecting a codon conserved across species. This variant is predicted likely pathogenic, according to ACMG criteria. It is absent from the ClinVar database (as of August 2020). A different missense mutation at the same amino acid position ( $p$. H481P) has been previously reported affecting that codon in a Swedish patient with T-B' SCID phenotype (51). RAG2-H481 is one of the zinc-binding residues in the PHD domain (Figure $\mathrm{S1C}$ ), in a $\mathrm{Cys}_{2}-\mathrm{His}_{2}$ binding site that lies directly below the dimethylated arginine residue of the histone-3 peptide. This suggests that correct assembly of this site is important for histone recognition and binding. Close inspection of nearby residues shows that RAG2-H448 and RAG2-E480 are also within range to participate in zinc binding (Figure 5A). Mutation to D generates a Cys ${ }_{2}$-His-Asp binding site (Figure 5B), which is also possible but much less frequent (52), and is probably a less avid binding site, given the lower affinity of carboxylates for zinc, compared to imidazole or thiolates (53). It was recently shown for a RanBP2-type zinc finger (54) that having nearby sidechains that can compete for zinc binding renders the complex kinetically unstable. This could be at work for this mutation, where residues 448,480 , and 481 would compete for zinc binding, wrecking the proper geometry of this binding site and of the histonebinding surface at the opposite side of the beta sheet. That zinc binding is important as shown by the fact that mutation of RAG2-C446 and C478 to W or Y, respectively, cause SCID (HMGD); only RAG2-C478Y is found in gnomAD, and tolerated as a heterozygote.

\section{Case 5}

The patient is a female from a non-consanguineous family who tolerated the BCG vaccine. Since 22 days of age, she suffered from diarrhea, receiving changes in infant formulas with no improvement. The patient was passing four to five daily stools, associated with the appearance of oral white ulcers with erythematous borders in oropharynx, inner checks under and under the tongue. She was treated with acyclovir without improvement. Subsequently, she developed a perianal abscess, urinary tract infection and anemia. She was hospitalized and treated with several intravenous antibiotic regimes. Laboratory work-up showed low levels of serum immunoglobulins and lymphopenia (Table S2). The patient received a haploidentical stem cell transplant at age 9 months old, and one year later she is in a good health with $100 \%$ of chimerism and full immune reconstitution. Whole-exome sequencing revealed a homozygous variant in exon 2 of RAG1, RAG1(NM_000448.3):c.2291G>C, (p.Arg764Pro) a position highly conserved across mammals, including humans and primates. This variant is predicted likely pathogenic by ACMG and it is absent of genomAD database. RAG1-R764 lies at the C-terminus of an alpha helix in RAG1, facing the second Kelch repeat of RAG2 (Figure S1A and S1B). It engages in a salt bridge and a hydrogen bond with RAG2-E126 and RAG2-Y108, respectively. Another salt bridge with RAG1-E761, in the preceding turn of the alpha helix, stabilizes the complex (Figure 6A). The Arg764Pro variant causes loss of all these 
interactions, and the additional loss of a backbone hydrogen bond between the amide $\mathrm{NH}$ group of residue 764 and the $\mathrm{CO}$ group of residue 760 in the alpha helix (Figure 6B). This mutation destabilizes both RAG1 and the complex with RAG2. It was described in a heterozygous patient that had three different mutations, two of which rendered inactive RAG (55). RAG1-R764P is not functional and is expressed at a lower level than the wild type (70\%), along the line of our structural interpretation. Regarding the residue neighbors, a mutation of RAG2-E126 to Q or to R is benign (gnomAD), indicating that other sidechains may fill in the gap left by this residue.

\section{Case 6}

A 3-month-old female infant was brought for a history of severe atopic dermatitis, chronic perforated otitis media and recurrent oral candidiasis. Family history was significant for consanguinity and early deaths where the proband's sister had died of septic shock following severe pneumonia. On physical examination, a ruptured eardrum was confirmed, together with extensive severe eczema, oral white plaques, and absent tonsils and lymph nodes. Omenn syndrome in the context of SCID was suspected, and the patient was started on oral ambulatory antibiotic prophylaxis, together with monthly IVIG. Laboratory work-up showed low levels of serum immunoglobulins and lymphopenia (Table S2). She underwent haploidentical HSCT, full chimerism and complete immune reconstitution was achieved.

Genetic diagnosis was confirmed through WES after the successful transplant: a deleterious homozygous missense variant in exon 2 of $R A G 1$ (c.1871G>A, R624H). It is registered in ClinVar, and predicted to be probably damaging by PolyPhen and deleterious with low confidence by SIFT. Of the six reported mutations of this work, this is the most frequent one in gnomAD ( 12 heterozygotes, from different parts of the world). This mutation has been reported previously $(5,41,56)$, as a variant with severe reduction in activity when assayed in vitro, but with comparable expression levels as the wild type (Table S2). The mutation is tolerated as heterozygous with a functional allele. R624 lies within a beta sheet that partially covers the alpha helix where one of the catalytic residues (E965) resides (Figure 7A). Its long sidechain allows the guanidinium nitrogens to engage in hydrogen bonds that stabilize the position of two catalytic residues (E965 and D603), via interactions with both the mainchain and sidechain of N964. Upon mutation to histidine, all these interactions are lost (Figure 7B), suggesting that the active site would have a different orientation, resulting in lower activity.

\section{Discussion}

We have described a series of six patients from unrelated families harboring missense pathogenic variants in recombinase genes: 2 in RAG1, and 4 in RAG2. Two of those variants in RAG2 (L155P and H481D) were not previously associated with SCID phenotypes. According to gnomAD database, these two variants has been only identified in Latino individuals. Three patients presented with a phenotype of Omenn syndrome, one with radiosensitive SCID, and two with CID. Both patients with RAG1 deficiencies were successfully transplanted during their first year and are currently alive and healthy; in contrast, three of the RAG2 deficient patients have died, and the last one is lost to follow-up. This is, to our knowledge, 
the first case series of RAG1 or RAG2 deficient patients from Mexico and Latin America. Although the sample size is small by any standard, we were able to show the wide clinical spectrum (Omenn, leaky and full-blown SCID), as well as the structural modeling and in silico analysis of the protein consequences for the different variants. Moreover, for the two pathogenic variants non previously associated with SCID variant, we were able to show an abnormal $\mathrm{T}$ cell function as evidenced by an impaired cell proliferation.

Biallelic missense germline variants in RAG1 and RAG2 could impair RAG function for many different reasons. Destabilizing the proteins would result in lower amounts of working enzyme, and these are made in scant amounts as it is (57). Weakening the interaction between RAG1 and RAG2 would also reduce the amount of working enzyme. While both RAG1 and RAG2 can bind DNA independently (58), RAG1 is 10 to 100 times less active in the absence of RAG2, and it does not follow the 12/23 rule, leading to aberrant recombination events (57). Recruiting RAG to active chromatin is the function of the PHD domain, and it also increases both DNA binding and catalysis (59). Five of the six patients described in this work have alterations in one or more of these processes: RAG1-R764P destabilizes RAG1; RAG2L155P, -R229W, and -H481D destabilize RAG2; RAG1-R764P, RAG2-G35V, and RAG2-R229W destabilize the RAG1-RAG2 complex; and RAG2-H481D destabilizes the interaction with histones. Another important reason for poor function is the inability to assemble the active site, which is the case of patient six. Other reasons for poor function are alterations in the contact surface with DNA, or in RAG1-RAG1 interactions, but as mutations in patients lie far from these sites (Supplementary Figure 1), we did not entertain these mechanisms as the reason for disease.

All six patients were diagnosed and treated in the three largest cities in Mexico. We need to improve the awareness, to increase the referrals of inborn errors of immunity, especially for SCID, a pediatric emergency. In Mexico and Latin America, a few regions are known to practice consanguineous marriages, although a more frequent occurrence is a high degree of inbreeding in closed, geographically or culturally isolated communities of less than 1,000 inhabitants. Known consanguinity, a family history of early deaths, severe adverse reactions to the BCG vaccine, and an early onset of recurrent infections, are all well-known red flags that should raise the suspicion for AR-SCID in general, and RAG deficiencies in particular. We have yet to implement newborn screening for SCID in our country, to identify patients with the most severe form of inborn errors of immunity during their first month of life, before infectious and non-infectious complications ensue, and the chances of a successful HSCT are highest.

In conclusion, here we presented six patients with RAG1/2 mutations. All were missense variants and, therefore, some residual recombinase $\mathrm{V}(\mathrm{D}) \mathrm{J}$ activity may have been present. For the case of $R A G 2$, three variants were in the core domain, and one in the plant homeodomain (PHD), whereas the variants identified for $R A G 1$, one was in the zinc-binding domain, and the other was adjacent to the catalytic site. As previously reported, the broad spectrum of phenotypes associated with RAG1/2 variants depends on the position of mutations and how these impact on recombinase $V(D) J$ activity. Consequently, information on repertoire diversity in $T$ lymphocytes and their phenotypes will be of interest to understand how RAG1/2 pathogenic variants may result in this broad spectrum of clinical manifestations, defining abnormal immunological responses such as inflammation and autoimmunity. 


\section{Declarations}

\section{Ethics Approval, Consent to Participate, and Consent for Publication}

This study and the written consent forms were approved by the local ethics committees in accordance with the declaration of Helsinki (Protocols 00165 and 049/2013).

\section{Acknowledgements}

The authors would like to thank the patients and their families for participating in this study.

\section{Contributions}

MECM and SEP conceived the study. MECM, SORL and NP wrote the manuscript. SORL, JN, SDR and LDN performed, analyzed, and interpreted the genetics. NP performed and analyzed the protein modeling. EGS and LBR performed proliferation assays and provided flow cytometry data. CAV, CMG, LLFL, and KCS provided the in silico analysis and classification of RAG1/2 variants. MAYN, SSM, GW, MENN, APMR, ATSB, EVM took care of the patients and provided clinical data. PG, JAY performed genetic studies. IMD provided their expertise on congenital disorders. All authors critically reviewed the manuscript and approved the final version.

\section{Funding}

MECM is supported by Consejo Nacional de Ciencia y Tecnología (CONACYT 281854).

LDN is supported by the Division of Intramural Research, National Institute of Allergy and Infectious Diseases, National Institutes of Health, Bethesda, MD, USA.

\section{Disclosure of conflicts of interest}

The authors declare that they have no conflicts of interest.

\section{References}

1. Sponzilli I, Notarangelo LD. Severe combined immunodeficiency (SCID): from molecular basis to clinical management. Acta Biomed. 2011;82(1):5-13.

2. Fischer A. Severe combined immunodeficiencies (SCID). Clin Exp Immunol. 2000;122(2):143-9.

3. Cossu F. Genetics of SCID. Ital J Pediatr. 2010;36:76.

4. Notarangelo LD, Kim MS, Walter JE, Lee YN. Human RAG mutations: biochemistry and clinical implications. Nat Rev Immunol. 2016;16(4):234-46.

5. Schwarz K, Gauss GH, Ludwig L, Pannicke U, Li Z, Lindner D, et al. RAG mutations in human B cellnegative SCID. Science. 1996;274(5284):97-9. 
6. Delmonte OM, Schuetz C, Notarangelo LD. RAG Deficiency: Two Genes, Many Diseases. J Clin Immunol. 2018;38(6):646-55.

7. De Ravin SS, Cowen EW, Zarember KA, Whiting-Theobald NL, Kuhns DB, Sandler NG, et al. Hypomorphic Rag mutations can cause destructive midline granulomatous disease. Blood. 2010;116(8):1263-71.

8. Patiroglu T, Akar HH, Gilmour K, Ozdemir MA, Bibi S, Henriquez F, et al. Atypical severe combined immunodeficiency caused by a novel homozygous mutation in Rag1 gene in a girl who presented with pyoderma gangrenosum: a case report and literature review. J Clin Immunol. 2014;34(7):792-5.

9. Schuetz C, Huck K, Gudowius S, Megahed M, Feyen O, Hubner B, et al. An immunodeficiency disease with RAG mutations and granulomas. N Engl J Med. 2008;358(19):2030-8.

10. Villa A, Notarangelo LD. RAG gene defects at the verge of immunodeficiency and immune dysregulation. Immunol Rev. 2019;287(1):73-90.

11. Berman HM, Westbrook J, Feng Z, Gilliland G, Bhat TN, Weissig H, et al. The Protein Data Bank. Nucleic Acids Res. 2000;28(1):235-42.

12. Kim MS, Lapkouski M, Yang W, Gellert M. Crystal structure of the V(D)J recombinase RAG1-RAG2. Nature. 2015;518(7540):507-11.

13. Kim MS, Chuenchor W, Chen X, Cui Y, Zhang X, Zhou ZH, et al. Cracking the DNA Code for V(D)J Recombination. Mol Cell. 2018;70(2):358-70 e4.

14. Chen $X$, Cui Y, Best RB, Wang H, Zhou ZH, Yang W, et al. Cutting antiparallel DNA strands in a single active site. Nat Struct Mol Biol. 2020;27(2):119-26.

15. Chen X, Cui Y, Wang H, Zhou ZH, Gellert M, Yang W. How mouse RAG recombinase avoids DNA transposition. Nat Struct Mol Biol. 2020;27(2):127-33.

16. Ru H, Chambers MG, Fu TM, Tong AB, Liao M, Wu H. Molecular Mechanism of V(D)J Recombination from Synaptic RAG1-RAG2 Complex Structures. Cell. 2015;163(5):1138-52.

17. Ru H, Mi W, Zhang P, Alt FW, Schatz DG, Liao M, et al. DNA melting initiates the RAG catalytic pathway. Nat Struct Mol Biol. 2018;25(8):732-42.

18. Zhang Y, Cheng TC, Huang G, Lu Q, Surleac MD, Mandell JD, et al. Transposon molecular domestication and the evolution of the RAG recombinase. Nature. 2019;569(7754):79-84.

19. Humphrey W, Dalke A, Schulten K. VMD: visual molecular dynamics. J Mol Graph. 1996;14(1):33-8, 27-8.

20. Russell RB, Barton GJ. Multiple protein sequence alignment from tertiary structure comparison: assignment of global and residue confidence levels. Proteins. 1992;14(2):309-23.

21. Roberts E, Eargle J, Wright D, Luthey-Schulten Z. MultiSeq: unifying sequence and structure data for evolutionary analysis. BMC Bioinformatics. 2006;7:382.

22. Bertoni M, Kiefer F, Biasini M, Bordoli L, Schwede T. Modeling protein quaternary structure of homoand hetero-oligomers beyond binary interactions by homology. Sci Rep. 2017;7(1):10480. 
23. Ramon-Maiques S, Kuo AJ, Carney D, Matthews AG, Oettinger MA, Gozani O, et al. The plant homeodomain finger of RAG2 recognizes histone $\mathrm{H} 3$ methylated at both lysine-4 and arginine-2. Proc Natl Acad Sci U S A. 2007;104(48):18993-8.

24. Jo S, Kim T, lyer VG, Im W. CHARMM-GUI: a web-based graphical user interface for CHARMM. J Comput Chem. 2008;29(11):1859-65.

25. Brooks BR, Brooks CL, 3rd, Mackerell AD, Jr., Nilsson L, Petrella RJ, Roux B, et al. CHARMM: the biomolecular simulation program. J Comput Chem. 2009;30(10):1545-614.

26. Lee J, Cheng X, Swails JM, Yeom MS, Eastman PK, Lemkul JA, et al. CHARMM-GUI Input Generator for NAMD, GROMACS, AMBER, OpenMM, and CHARMM/OpenMM Simulations Using the CHARMM36 Additive Force Field. J Chem Theory Comput. 2016;12(1):405-13.

27. Lovell SC, Davis IW, Arendall WB, 3rd, de Bakker PI, Word JM, Prisant MG, et al. Structure validation by Calpha geometry: phi,psi and Cbeta deviation. Proteins. 2003;50(3):437-50.

28. Richards S, Aziz N, Bale S, Bick D, Das S, Gastier-Foster J, et al. Standards and guidelines for the interpretation of sequence variants: a joint consensus recommendation of the American College of Medical Genetics and Genomics and the Association for Molecular Pathology. Genet Med. 2015;17(5):405-24.

29. Adzhubei IA, Schmidt S, Peshkin L, Ramensky VE, Gerasimova A, Bork P, et al. A method and server for predicting damaging missense mutations. Nat Methods. 2010;7(4):248-9.

30. Sim NL, Kumar P, Hu J, Henikoff S, Schneider G, Ng PC. SIFT web server: predicting effects of amino acid substitutions on proteins. Nucleic Acids Res. 2012;40(Web Server issue):W452-7.

31. Bendl J, Stourac J, Salanda O, Pavelka A, Wieben ED, Zendulka J, et al. PredictSNP: robust and accurate consensus classifier for prediction of disease-related mutations. PLoS Comput Biol. 2014;10(1):e1003440.

32. Laskowski RA, Stephenson JD, Sillitoe I, Orengo CA, Thornton JM. VarSite: Disease variants and protein structure. Protein Sci. 2020;29(1):111-9.

33. Ioannidis NM, Rothstein JH, Pejaver V, Middha S, McDonnell SK, Baheti S, et al. REVEL: An Ensemble Method for Predicting the Pathogenicity of Rare Missense Variants. Am J Hum Genet. 2016;99(4):877-85.

34. Niroula A, Urolagin S, Vihinen M. PON-P2: prediction method for fast and reliable identification of harmful variants. PLoS One. 2015;10(2):e0117380.

35. Banerjee A, Mitra P. Estimating the Effect of Single-Point Mutations on Protein Thermodynamic Stability and Analyzing the Mutation Landscape of the p53 Protein. J Chem Inf Model. 2020;60(6):3315-23.

36. Ittisoponpisan S, Islam SA, Khanna T, Alhuzimi E, David A, Sternberg MJE. Can Predicted Protein 3D Structures Provide Reliable Insights into whether Missense Variants Are Disease Associated? J Mol Biol. 2019;431(11):2197-212.

37. Meshaal SS, El Hawary RE, Abd Elaziz DS, Eldash A, Alkady R, Lotfy S, et al. Phenotypical heterogeneity in RAG-deficient patients from a highly consanguineous population. Clin Exp Immunol. 
2019;195(2):202-12.

38. Lev A, Simon AJ, Trakhtenbrot L, Goldstein I, Nagar M, Stepensky P, et al. Characterizing T cells in SCID patients presenting with reactive or residual T lymphocytes. Clin Dev Immunol. 2012;2012:261470.

39. Sobacchi C, Marrella V, Rucci F, Vezzoni P, Villa A. RAG-dependent primary immunodeficiencies. Hum Mutat. 2006;27(12):1174-84.

40. Tabori U, Mark Z, Amariglio N, Etzioni A, Golan H, Biloray B, et al. Detection of RAG mutations and prenatal diagnosis in families presenting with either T-B- severe combined immunodeficiency or Omenn's syndrome. Clin Genet. 2004;65(4):322-6.

41. Corneo B, Moshous D, Gungor T, Wulffraat N, Philippet P, Le Deist FL, et al. Identical mutations in RAG1 or RAG2 genes leading to defective $V(D) J$ recombinase activity can cause either T-B-severe combined immune deficiency or Omenn syndrome. Blood. 2001;97(9):2772-6.

42. Karczewski KJ, Francioli LC, Tiao G, Cummings BB, Alfoldi J, Wang Q, et al. The mutational constraint spectrum quantified from variation in 141,456 humans. Nature. 2020;581(7809):434-43.

43. Sherry ST, Ward MH, Kholodov M, Baker J, Phan L, Smigielski EM, et al. dbSNP: the NCBI database of genetic variation. Nucleic Acids Res. 2001;29(1):308-11.

44. Cooper DN, Ball EV, Krawczak M. The human gene mutation database. Nucleic Acids Res. 1998;26(1):285-7.

45. Luk ADW, Lee PP, Mao H, Chan KW, Chen XY, Chen TX, et al. Family History of Early Infant Death Correlates with Earlier Age at Diagnosis But Not Shorter Time to Diagnosis for Severe Combined Immunodeficiency. Front Immunol. 2017;8:808.

46. Al-Mousa H, Abouelhoda M, Monies DM, Al-Tassan N, Al-Ghonaium A, Al-Saud B, et al. Unbiased targeted next-generation sequencing molecular approach for primary immunodeficiency diseases. $\mathrm{J}$ Allergy Clin Immunol. 2016;137(6):1780-7.

47. Schuetz C, Neven B, Dvorak CC, Leroy S, Ege MJ, Pannicke U, et al. SCID patients with ARTEMIS vs RAG deficiencies following HCT: increased risk of late toxicity in ARTEMIS-deficient SCID. Blood. 2014;123(2):281-9.

48. Safaei S, Pourpak Z, Moin M, Houshmand M. IL7R and RAG1/2 genes mutations/polymorphisms in patients with SCID. Iran J Allergy Asthma Immunol. 2011;10(2):129-32.

49. Villa A, Sobacchi C, Notarangelo LD, Bozzi F, Abinun M, Abrahamsen TG, et al. V(D)J recombination defects in lymphocytes due to RAG mutations: severe immunodeficiency with a spectrum of clinical presentations. Blood. 2001;97(1):81-8.

50. Gomez CA, Ptaszek LM, Villa A, Bozzi F, Sobacchi C, Brooks EG, et al. Mutations in conserved regions of the predicted RAG2 kelch repeats block initiation of $\mathrm{V}(\mathrm{D}) \mathrm{J}$ recombination and result in primary immunodeficiencies. Mol Cell Biol. 2000;20(15):5653-64.

51. Noordzij JG, de Bruin-Versteeg S, Verkaik NS, Vossen JM, de Groot R, Bernatowska E, et al. The immunophenotypic and immunogenotypic B-cell differentiation arrest in bone marrow of RAG- 
deficient SCID patients corresponds to residual recombination activities of mutated RAG proteins. Blood. 2002;100(6):2145-52.

52. Ireland SM, Martin ACR. ZincBind-the database of zinc binding sites. Database (Oxford). 2019;2019.

53. Krezel A, Maret W. The biological inorganic chemistry of zinc ions. Arch Biochem Biophys. 2016;611:3-19.

54. Soni K, Martinez-Lumbreras S, Sattler M. Conformational Dynamics from Ambiguous Zinc Coordination in the RanBP2-Type Zinc Finger of RBM5. J Mol Biol. 2020;432(14):4127-38.

55. Lee YN, Frugoni F, Dobbs K, Walter JE, Giliani S, Gennery AR, et al. A systematic analysis of recombination activity and genotype-phenotype correlation in human recombination-activating gene 1 deficiency. J Allergy Clin Immunol. 2014;133(4):1099-108.

56. Alsmadi O, Al-Ghonaium A, Al-Muhsen S, Arnaout R, Al-Dhekri H, Al-Saud B, et al. Molecular analysis of T-B-NK+ severe combined immunodeficiency and Omenn syndrome cases in Saudi Arabia. BMC Med Genet. 2009;10:116.

57. Carmona LM, Fugmann SD, Schatz DG. Collaboration of RAG2 with RAG1-like proteins during the evolution of V(D)J recombination. Genes Dev. 2016;30(8):909-17.

58. Oudinet C, Braikia FZ, Dauba A, Khamlichi AA. Recombination may occur in the absence of transcription in the immunoglobulin heavy chain recombination centre. Nucleic Acids Res. 2020;48(7):3553-66.

59. Shimazaki N, Tsai AG, Lieber MR. H3K4me3 stimulates the V(D)J RAG complex for both nicking and hairpinning in trans in addition to tethering in cis: implications for translocations. Mol Cell. 2009;34(5):535-44.

\section{Figures}




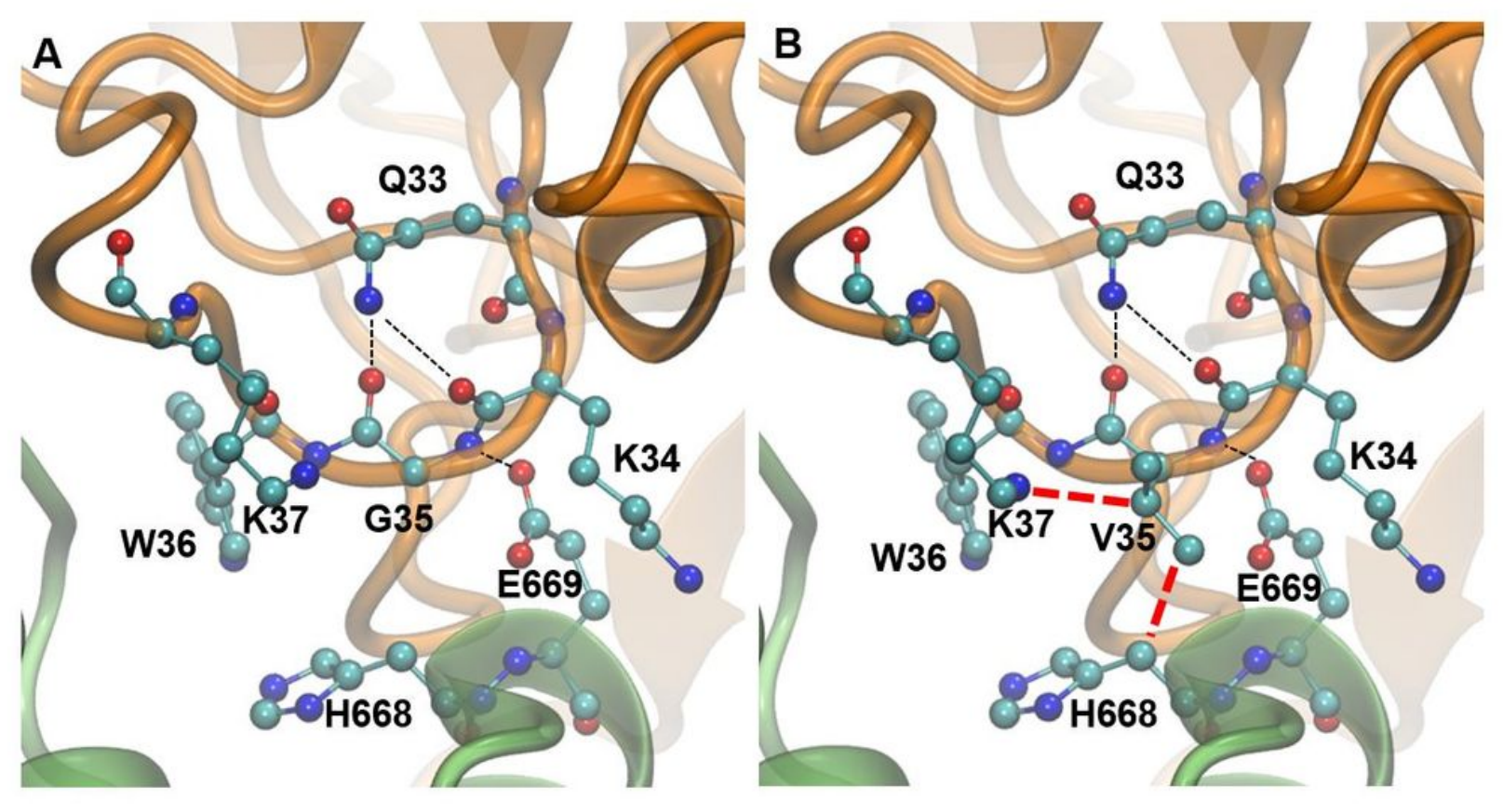

Fig 1

Figure 1

Effect of the G35V mutation. RAG1 is shown as green ribbon and RAG2 in orange, approximately in the same orientation as Supplementary Figure 1A; all residues are shown in balls and sticks and CPK colors (carbon in cyan, nitrogen in blue, oxygen in red). (A) shows the wild type structure, without steric clashes and a network of hydrogen bonds (broken black lines) between the sidechain of Q33 and the backbone carbonyls of G35 and K34, ensuring the right conformation for the interaction of E669 with the backbone amide of G35. (B) shows the sidechain of V35, engaging in potential clashes (broken red lines) with H668, K37 and its backbone atoms. These clashes cannot be relieved by rotating the sidechain, which would result in a close contact with W36. 


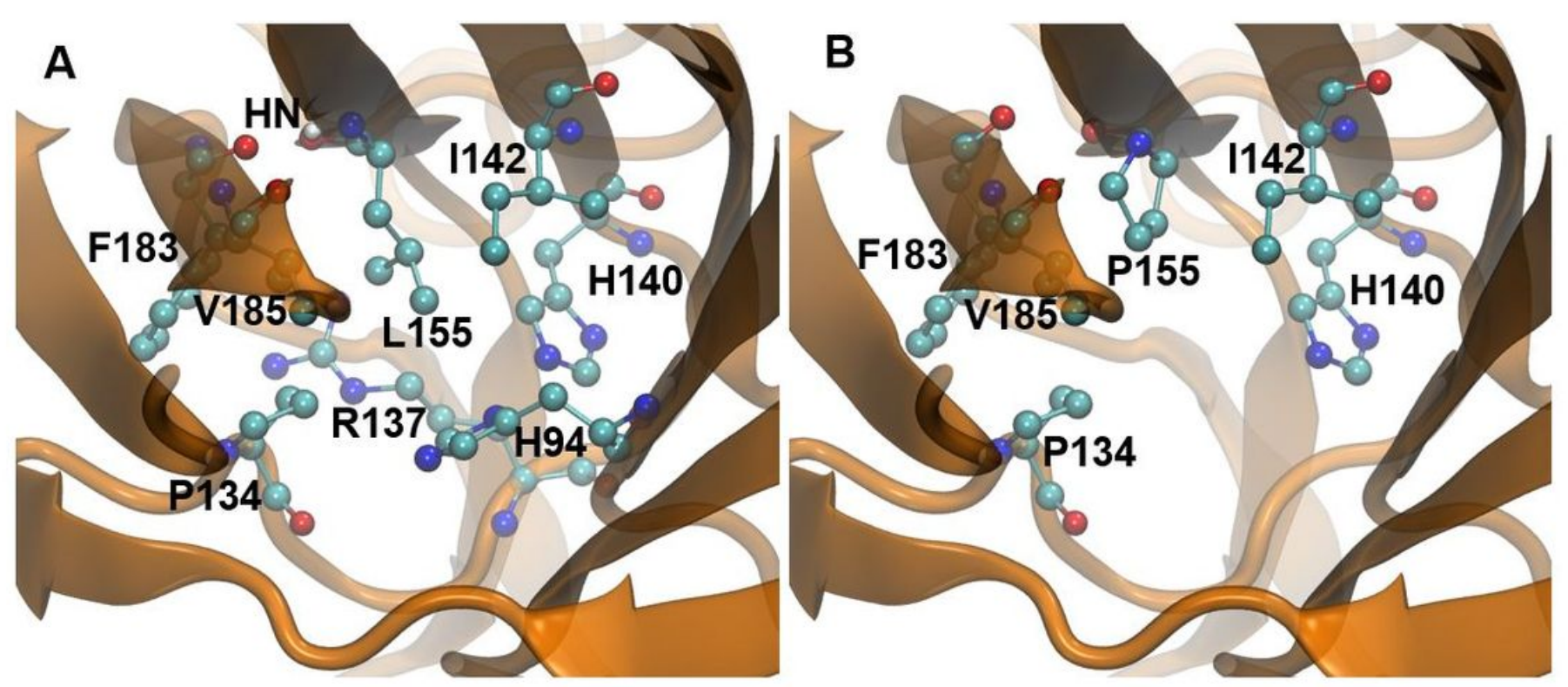

Figure 2

Effect of the L155P mutation. RAG2 is shown as an orange ribbon, seen from the top of Supplementary Figure 1A; all residues are shown in balls and sticks and CPK colors (carbon in cyan, nitrogen in blue, oxygen in red). (A) shows the hydrophobic and aromatic cage around L155, with interactions that bridge three beta strands in the Kelch repeat and link it to the previous one (through H94). Also, a backbone hydrogen bond, indicated by HN, binds L155 to F183 in the adjacent beta strand. (B) depicts the incomplete cage around P155, lacking H94 and R137, effectively losing interactions with the previous Kelch repeat. Also, the hydrogen bond to F183 is missing, leading to a weakened beta sheet. 

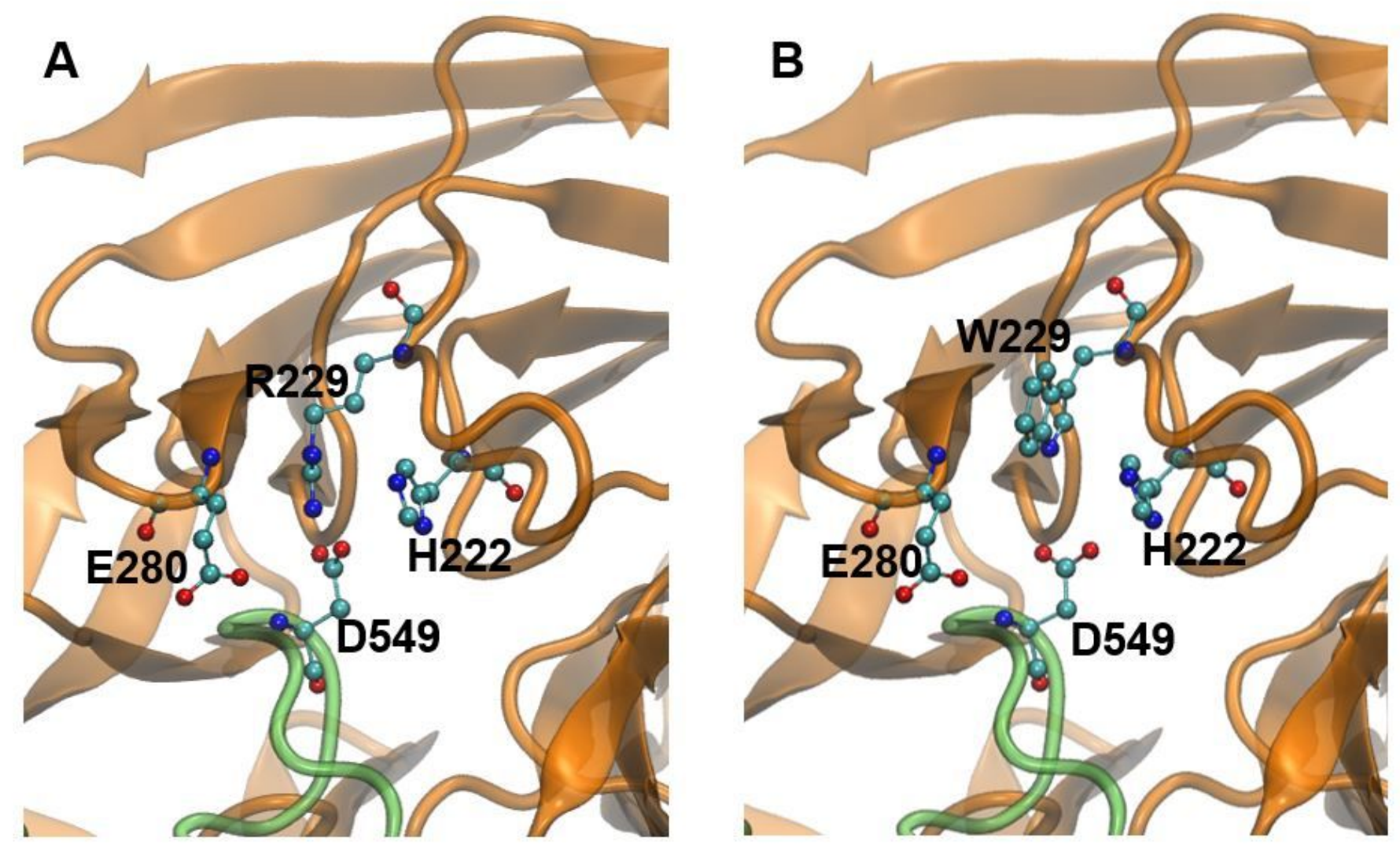

Figure 3

Effect of the R229W mutation. RAG1 is shown as a green ribbon and RAG2 in orange, looking at the bottom of RAG2 from the perspective of RAG1; all residues are shown in balls and sticks and CPK colors (carbon in cyan, nitrogen in blue, oxygen in red). (A) shows the structural role of R229 by interacting with adjacent residues of RAG2 from the previous Kelch repeat (H222) and its own repeat (E280), and its interaction with RAG1 (D549). 
A
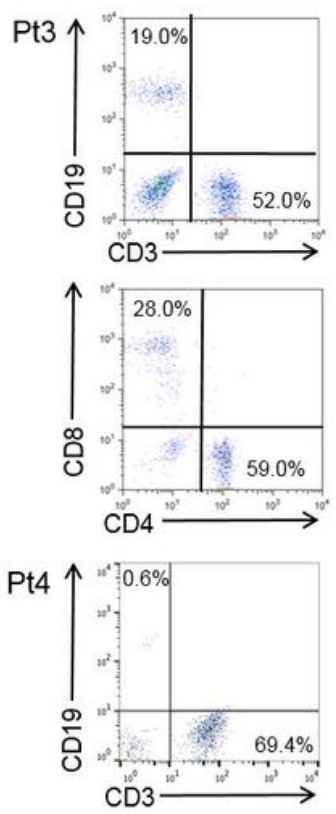

Fig 4

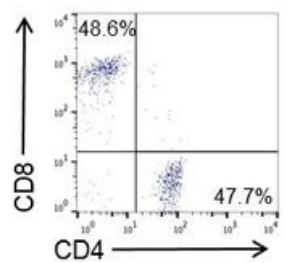

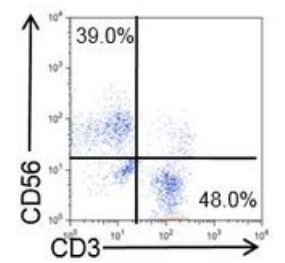
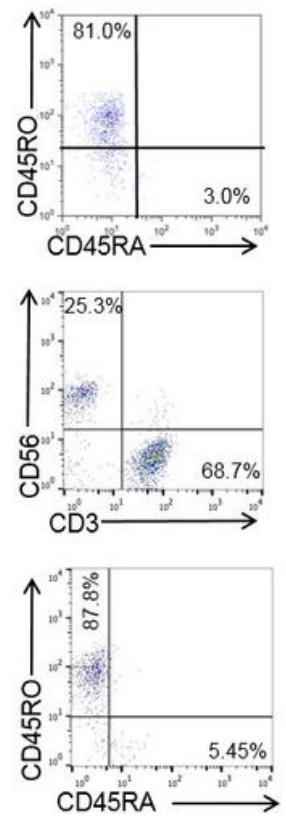

B

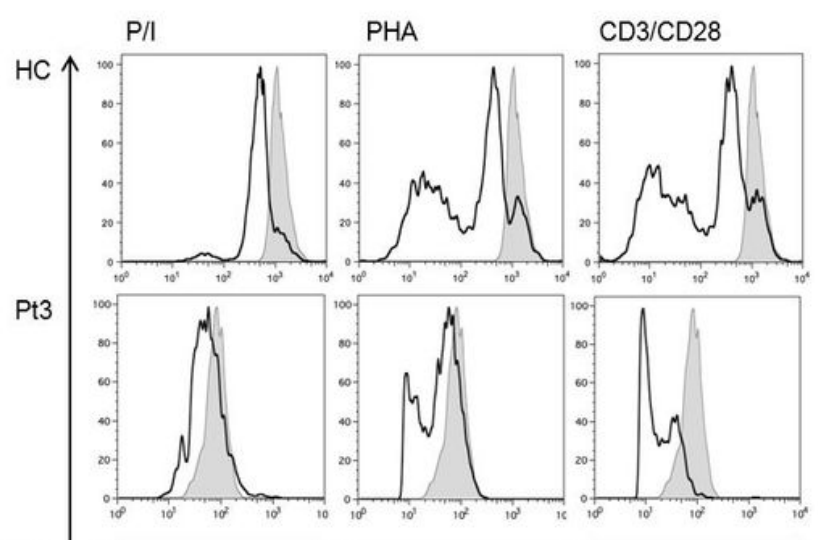

$\mathrm{HC}$

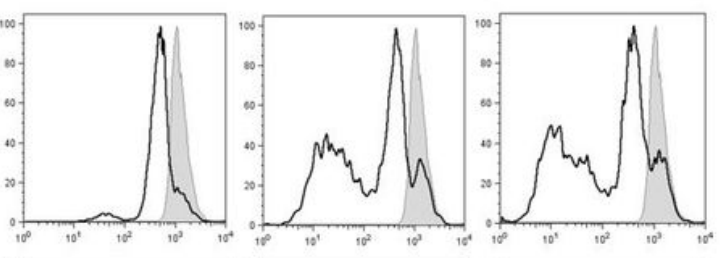

$\mathrm{P}+4$

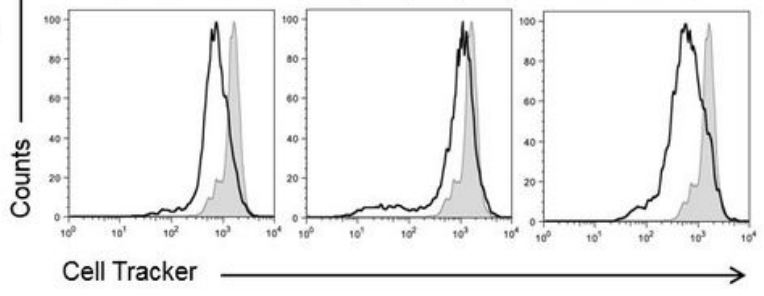

Figure 4

(A) PBMCs were stained with the following panel of fluorochrome-conjugated monoclonal Abs directed against following cell surface markers: CD3 FITC, CD4 PE, CD8 APC, CD19 PerCP-Cy5, CD56 APC, CD45 RA PE, and CD45 RO APC. Then cells were acquired on FACSCanto II (BD bioscience) and analyzed with the use of FlowJo 7.6.5 software (Tree Star, Ashland, OR). (B) PBMCs were subject to proliferation assays by staining PBMCs with CellTrackerTM green CMDFA dye (ThermoFisher Scientific, USA), then cells were stimulated with LEAF purified anti-CD3 plus anti-CD28 antibodies (BioLegend, USA) or PHA (Sigma) for 5 days at $370 \mathrm{C} 5 \mathrm{CO} 2$. Then, PBMCs were stained using the murine PE conjugated monoclonal antibody directed against human CD3. Flow cytometric data were acquired on FACSCanto II (BD bioscience) and analyzed with the use of FlowJo 7.6.5 software (Tree Star, Ashland, OR). Gates were set to include CD3+ lymphocytes. Thereafter, T cells were defined by the expression of CD3. 

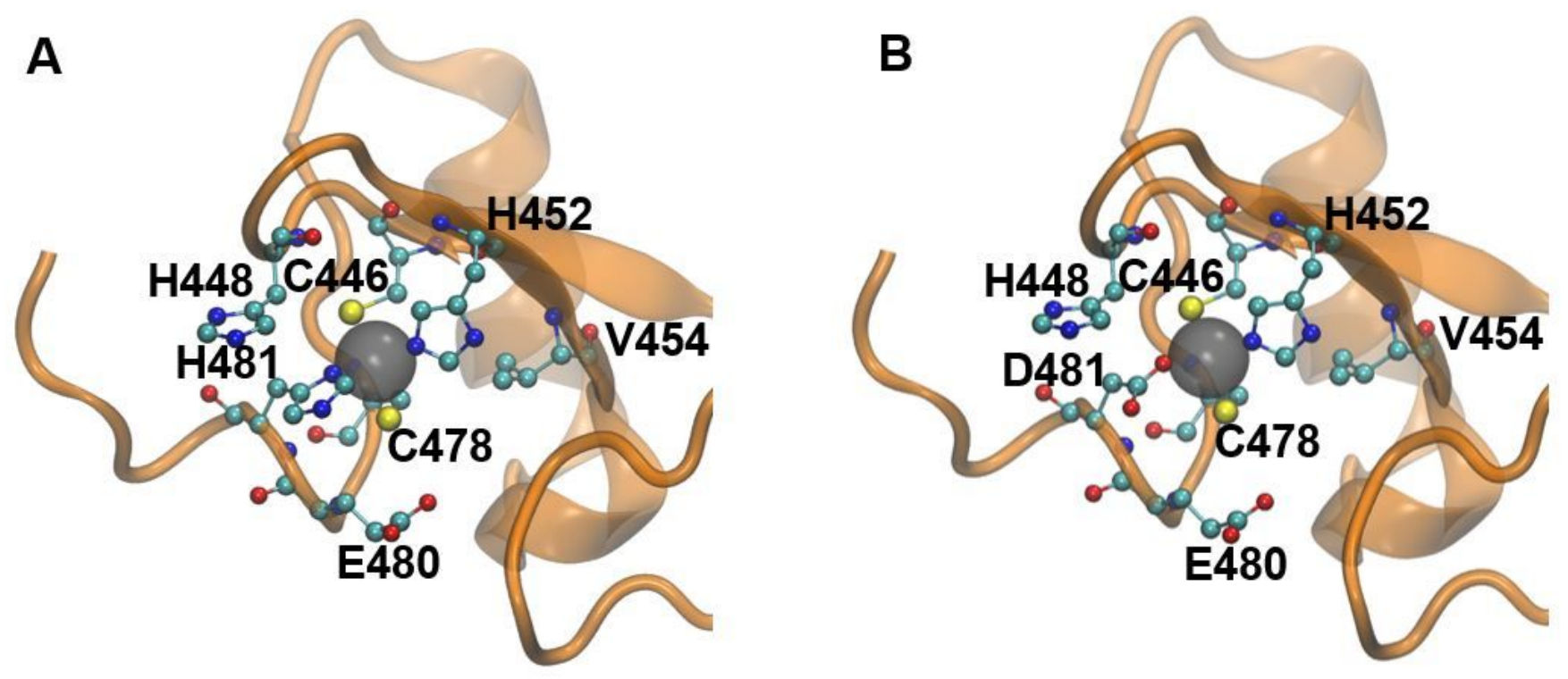

Figure 5

Effect of the H481D mutation. RAG2 is shown as an orange ribbon, in the same orientation as Supplementary Figure 1C. The coordination sphere of $\mathrm{Zn} 2$ is shown in balls and sticks; all residues are shown in CPK colors (carbon in cyan, nitrogen in blue, oxygen in red, sulfur in yellow). (A) shows the wild type environment of Zn2 and (B) shows the mutation to D481. Noteworthy are the neighboring histidine and glutamate residues, which could compete with D481 for coordinating Zn, and make this site kinetically labile.
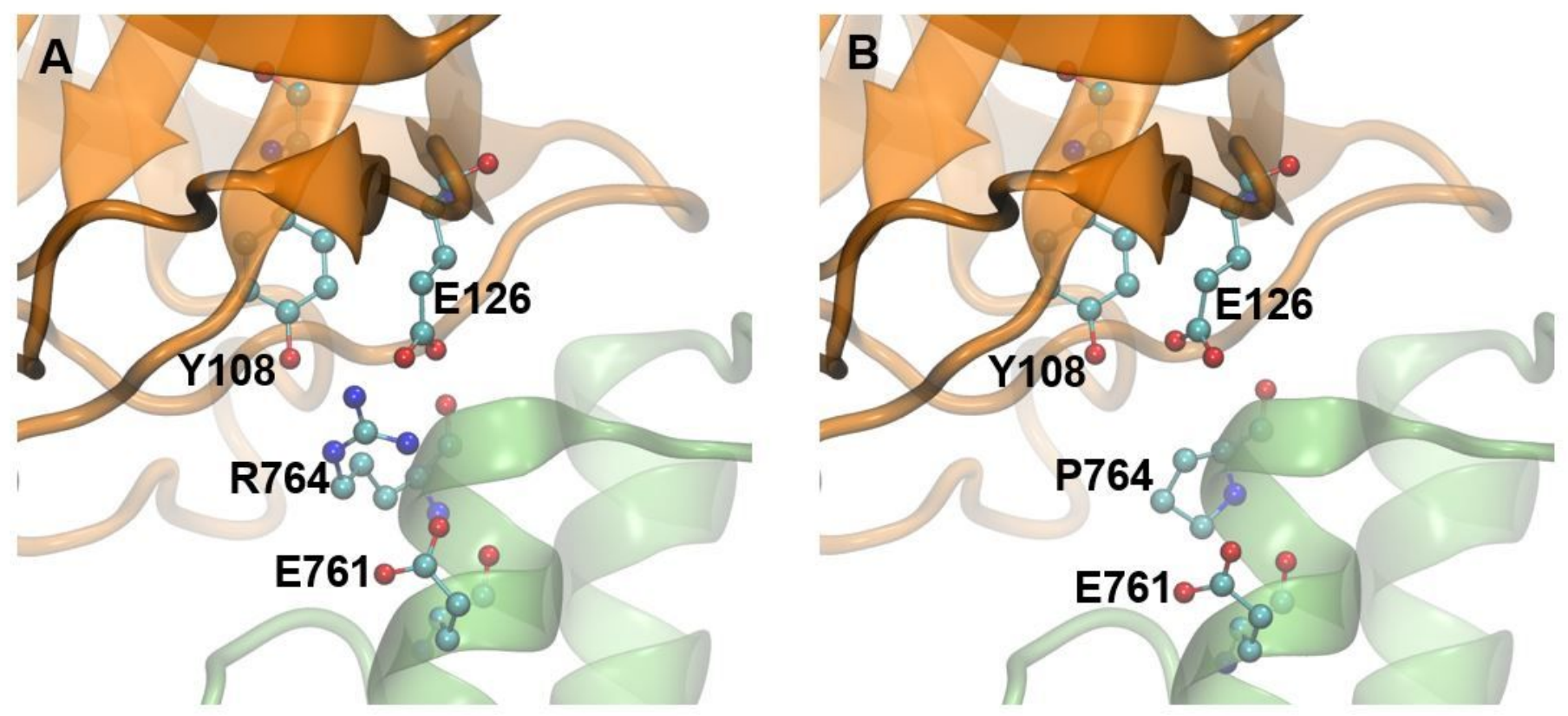

Figure 6 
Effect of the R764P mutation. RAG1 is shown as a green ribbon and RAG2 in orange, roughly in the same perspective as Supplementary Figure 1A; all residues are shown in balls and sticks and CPK colors (carbon in cyan, nitrogen in blue, oxygen in red). (A) shows the hydrogen bond and electrostatic interactions of R764 with Y108 and E126 in RAG2, and E761 one turn below in the alpha helix in RAG1, buttressing the conformation of the sidechain of R764. (B) shows that P764 no longer interacts with any of these sidechains and has also lost the possibility to form a hydrogen bond with its backbone imide, weakening the last turn of the helix.
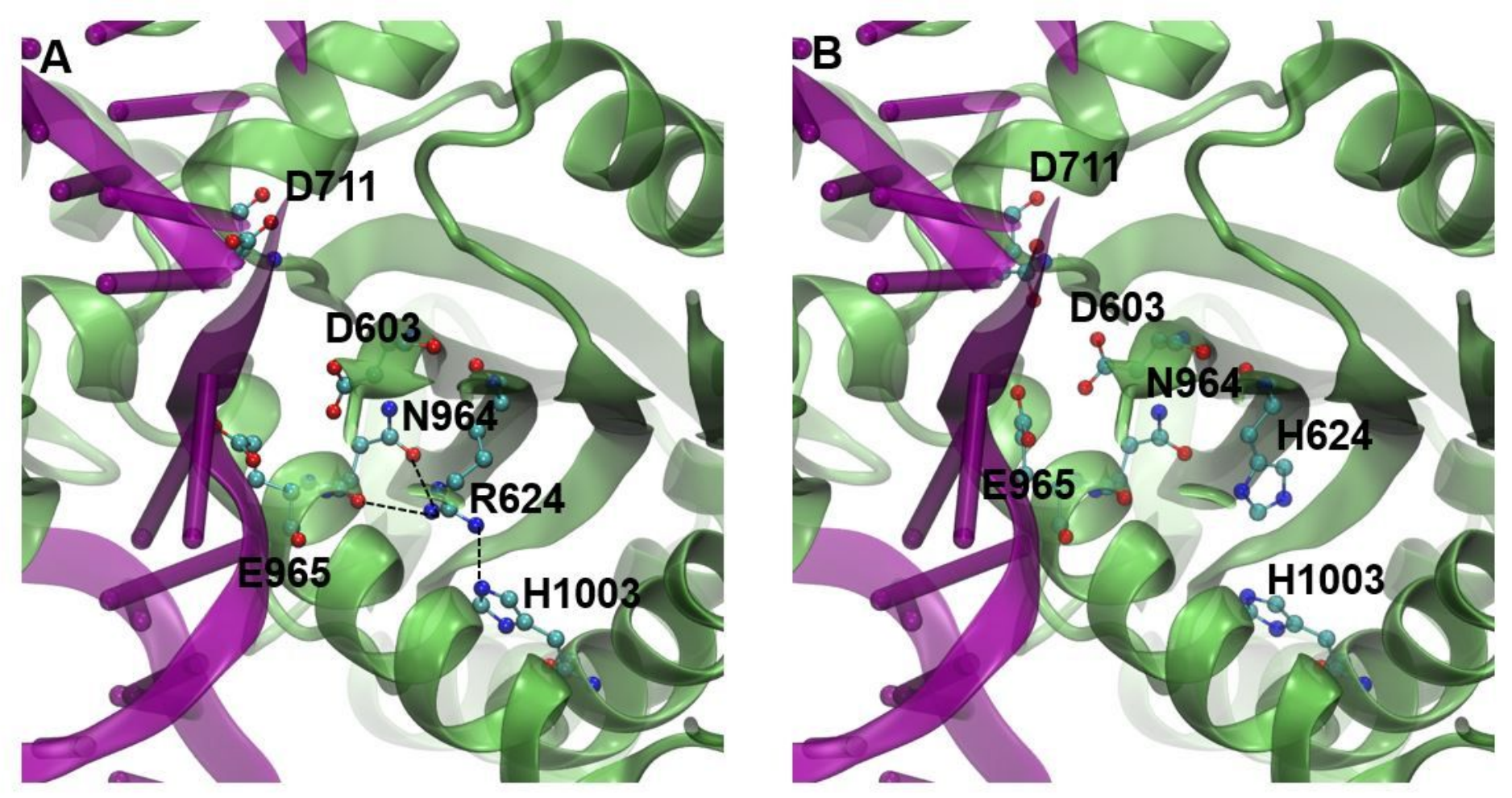

\section{Figure 7}

Effect of the R624H mutation. RAG1 is shown as a green ribbon and DNA in purple, zooming in at the active site; all residues are shown in balls and sticks and CPK colors (carbon in cyan, nitrogen in blue, oxygen in red). Residues D603, D711 and E965 constitute the active site residues, flanking the nick in the DNA strand. Hydrogen bonds are depicted as black broken lines. (A) shows the Hydrogen bond and electrostatic interactions of R624 with both the mainchain carbonyl and the sidechain carbonyl of N964, buttressing the position of the helix where E965 resides. R624 also engages in a Hydrogen bond with H1003 from an adjacent helix. N964 could also engage in favorable electrostatic interactions with D603. (B) H624, on account of being shorter, can no longer engage in any of the critical Hydrogen bonds that stabilize the position of the helix that forms part of the active site.

\section{Supplementary Files}

This is a list of supplementary files associated with this preprint. Click to download. 
- SupplementalData.pdf

Page 22/22 